This item was submitted to Loughborough's Research Repository by the author.

Items in Figshare are protected by copyright, with all rights reserved, unless otherwise indicated.

\title{
Exponential instability in an inverse problem for the Schrodinger equation
}

PLEASE CITE THE PUBLISHED VERSION

LICENCE

CC BY-NC-ND 4.0

\section{REPOSITORY RECORD}

Mandache, N.. 2019. "Exponential Instability in an Inverse Problem for the Schrodinger Equation”. figshare. https://hdl.handle.net/2134/680. 


\title{
Exponential instability in an inverse problem for Schrödinger equation
}

\author{
Niculae Mandache \\ University of Loughborough, Department of Mathematical Sciences, \\ Loughborough, LE11 3TU. E-mail: N.P.Mandache@lboro.ac.uk \\ On leave from: Institute of Mathematics, Romanian Academy, P.O. Box \\ 1-764, 70700 Bucharest, Romania. E-mail: mandache@stoilow.imar.ro
}

\begin{abstract}
We consider the problem of the determination of the potential from the Dirichlet to Neumann map of the Schrödinger operator. We show that this problem is severely ill posed. The results extend to the electrical impedance tomography. They show that the logarithmic stability results of Alessandrini are optimal.
\end{abstract}

\section{Introduction}

Let $\Omega \subset \mathbb{R}^{d}$ be a bounded domain with smooth boundary. We suppose $d \geq 2$. Consider the boundary value problem

$$
\left\{\begin{array}{l}
\left.u\right|_{\partial \Omega}=f \\
(-\Delta+q) u=0 \text { in } \Omega .
\end{array}\right.
$$

We suppose that $q$ is bounded and 0 is not a Dirichlet eigenvalue for $-\Delta+q$. This implies that the above system has a unique solution $u \in H^{1}(\Omega)$ for any

2000 Mathematics Subject Clasiffication: 35R30 (35J10,35J25) 
$f \in H^{1 / 2}(\partial \Omega)$. Then the Dirichlet to Neumann operator $\Lambda_{q}$ is defined by taking the exterior normal boundary derivative of $u: \Lambda_{q} f=\left.\frac{\partial u}{\partial \nu}\right|_{\partial \Omega}$.

We shall consider the problem of retrieving $q$ from $\Lambda_{q}$. It is related to the electrical impedance tomography, namely, determining the isotropic electrical conductivity $\gamma$ of an object, from measurements at its boundary. More precisely, we want to retrieve $\gamma$ from the voltage to current map $\tilde{\Lambda}_{\gamma}$ defined by: $\tilde{\Lambda}_{\gamma} f=\left.\gamma \frac{\partial v}{\partial \nu}\right|_{\partial \Omega}$, with

$$
\left\{\begin{array}{l}
\left.v\right|_{\partial \Omega}=f \\
\operatorname{div} \gamma \nabla v=0 \text { in } \Omega .
\end{array}\right.
$$

There is a standard reduction of the inverse problem for (2) to the inverse problem for (1). Indeed, taking $u=\gamma^{1 / 2} v$, we obtain that $(-\Delta+q) u=0$, with $q=\gamma^{-1 / 2} \Delta \gamma^{1 / 2}$ and we get $\Lambda_{q}=\gamma^{-1 / 2}\left(\tilde{\Lambda}_{\gamma} \gamma^{-1 / 2}+\frac{\partial \gamma^{1 / 2}}{\partial \nu}\right)$. This requires, however, to determine the restriction of $\gamma$ and of its normal derivative to the boundary first.

The inverse problem of electrical impedance tomography has a long history, most of the work stemming from Calderon's idea [7]. The unique determination of $\gamma$ and its derivatives at the boundary was obtained by Kohn and Vogelius [9] and stability by Sylvester and Uhlmann [15]. Global uniqueness in dimension $d \geq 3$, was proved for $\gamma \in C^{2}$ by Sylvester and Uhlmann [14]. In dimension 2, it was first proved by Nachman [12] for $\gamma \in W^{2, p}, p>1$, and then by Brown and Uhlmann [5] for $\gamma \in W^{1, p}, p>2$.

For the inverse problem for the Schrödinger operator with a smooth potential, in dimension greater than two, uniqueness is a consequence of the results in [14]. Nachman [11] extended this results to potentials in $L^{d / 2}$. In dimension 2 , uniqueness is known for small or generic potentials (i.e., for a dense open set of pairs of potentials in $W^{1, \infty} \times W^{1, \infty}$, Sun and Uhlmann [13]). The general case is open.

The stability in the problem (2) was obtained by Alessandrini in [1], [2] in dimension $d \geq 3$, using the complex geometric optics solutions of [14]. In dimension two, Liu [10] obtained stability for $\gamma \in C^{2}$ and Barceló, Barceló and Ruiz [6] for $\gamma \in C^{1+\varepsilon}$, using the approach of [5]. Suppose $1 / M<\gamma_{i}<M$ in 
$\Omega, i=1,2$, and $\left\|\gamma_{i}\right\|_{C^{2}} \leq M$. Then there are $\alpha>0$ and $C(M)>0$ such that

$$
\left\|\gamma_{1}-\gamma_{2}\right\|_{L^{\infty}} \leq C(M)\left(\log \left(1+\left\|\tilde{\Lambda}_{\gamma_{1}}-\tilde{\Lambda}_{\gamma_{2}}\right\|_{H^{1 / 2} \rightarrow H^{-1 / 2}}^{-1}\right)\right)^{-\alpha}
$$

Using the method in [3], one can show that (3) holds if $d \geq 3$ and $0<\alpha<2 / d$. See also Isakov [8], Theorem 5.2.3.

A similar result holds for (1). For any $d \geq 3$ and $m>0$, there is an $\alpha>0$, such that for every $M>0$ there is $C(M)>0$, so that $\left\|q_{i}\right\|_{C^{m}} \leq M, i=1,2$ implies

$$
\left\|q_{1}-q_{2}\right\|_{L^{\infty}} \leq C(M)\left(\log \left(1+\left\|\Lambda_{q_{1}}-\Lambda_{q_{2}}\right\|_{H^{1 / 2} \rightarrow H^{-1 / 2}}^{-1}\right)\right)^{-\alpha} .
$$

In fact, one way to obtain (3) is using (4) and the above reduction of (2) to (1). This was the approach of Sylvester and Uhlmann [14] for uniqueness and the same way was followed for stability for $d \geq 3$.

We show that the estimate (4) is optimal, in the sense that it cannot hold with $\alpha>m(2 d-1) / d$. The same restriction holds for $(3)$, if we suppose $\gamma_{i}$ bounded in $C^{m}, m \geq 2$. Similar instability results were obtained by Alessandrini [4] for the reconstruction of a part of the boundary that is unknown.

The paper is organised as follows. In the second section we give the notations, we make some conventions and state the main results. In the third section we prove a basic estimate for potentials supported strictly inside $\Omega$. In the fourth section we prove the main result, using a ball packing and covering by balls argument. In the final section we give an explicit, complex valued counterexample, discuss the case of radial potentials and make some remarks.

\section{Main results}

Conventions and notations. In what follows we fix $\Omega=B(0,1)$, the open unit ball in $\mathbb{R}^{d}$. If $m>0$ is not an integer we denote by $C^{m}$ the space $C^{[m],\{m\}}$, with $[m]$ and $\{m\}$ the integer part and the fractional part of $m$. We remind that $C^{k, \alpha}$ is the space of $C^{k}$ functions, with $\alpha$-Hölder continuous derivatives of order $k . C_{0}^{m}\left(\Omega^{\prime}\right)$ is the set of functions $\phi \in C^{m}$ with compact 
support $\operatorname{supp} \phi \subset \Omega^{\prime}$. We will use $C$ as a generic positive constant (different from formula to formula - we sometimes use $C^{\prime}, C^{\prime \prime}$, etc, to emphasize this). It may depend upon the dimension $d$, the order $m$, and the number $s$ (see Theorem 1). We fix an orthonormal basis $\left\{f_{j p}: j \geq 0,1 \leq p \leq p_{j}\right\}$ in $L^{2}\left(S^{d-1}\right)=L^{2}(\partial \Omega)$, with $f_{j p}$ spherical harmonic of degree $j$. Here $p_{j}=\left(\begin{array}{c}j+d-1 \\ d-1\end{array}\right)-\left(\begin{array}{c}j+d-3 \\ d-1\end{array}\right)$ is the dimension of the space of spherical harmonics of order $j$; we have $p_{j} \leq 2(j+1)^{d-2}$. In the Sobolev spaces $H^{s}\left(S^{d-1}\right)$ we will use the norm $\left\|\sum_{j, p} a_{j p} f_{j p}\right\|_{H^{s}}^{2}=\sum_{j, p}(1+j)^{2 s}\left|a_{j p}\right|^{2}$. The notation $\left(a_{j p k q}\right)$ stands for a multiple sequence (we drop the subscript $0 \leq j, 1 \leq p \leq p_{j}, 0 \leq k, 1 \leq q \leq p_{k}$ ). $|A|$ is the cardinality of a set $A$. When associating an operator in $L^{2}(\Omega)$ to a formal differential operator, we always assume the Dirichlet boundary conditions. We use polar coordinates $(r, \omega) \in \mathbb{R}_{+} \times S^{d-1}$, with $x=r \omega$. We denote by $\|A\|_{H S}$ the Hilbert-Schmidt norm of the matrix or operator $A$.

Theorem 1 For any $m>0$, any integer $d \geq 2$ and any $s \geq 0$, there is a constant $\beta>0$, such that for any $\varepsilon \in(0,1)$ there are real-valued potentials $q_{1}, q_{2} \in C^{m}$, such that

$$
\left\{\begin{array}{l}
\left\|\Lambda_{q_{1}}-\Lambda_{q_{2}}\right\|_{H^{-s} \rightarrow H^{s}} \leq \exp \left(-\varepsilon^{-\frac{d}{(2 d-1) m}}\right) \\
\left\|q_{1}-q_{2}\right\|_{\infty} \geq \varepsilon \\
\left\|q_{i}\right\|_{C^{m}} \leq \beta, i=1,2 \\
\left\|q_{i}\right\|_{\infty} \leq \varepsilon, \quad i=1,2
\end{array}\right.
$$

Corollary For any $m \geq 2, d \geq 2, s \geq 0$, there is a constant $\beta>0$ such that for any $\varepsilon \in(0,1)$ there are conductivities $\gamma_{1}, \gamma_{2} \in C^{m}$, with $1 \leq \gamma_{i} \leq 2$, such that

$$
\left\{\begin{array}{l}
\left\|\tilde{\Lambda}_{\gamma_{1}}-\tilde{\Lambda}_{\gamma_{2}}\right\|_{H^{-s} \rightarrow H^{s}} \leq \exp \left(-\varepsilon^{-\frac{d}{(2 d-1) m}}\right) \\
\left\|\gamma_{1}-\gamma_{2}\right\|_{\infty} \geq \varepsilon \\
\left\|\gamma_{i}\right\|_{C^{m}} \leq \beta, i=1,2
\end{array}\right.
$$

Remark We can allow $\beta$ to be arbitrarily small both in Theorem 1 and its corollary, if we require $\varepsilon \leq \varepsilon_{0}$ and replace the right hand side by $\exp \left(-c \varepsilon^{-\frac{d}{(2 d-1) m}}\right)$, with $\varepsilon_{0}>0$ and $c>0$ depending on $\beta$. 


\section{The basic estimate}

Lemma 1 Let $r_{0} \in(0,1)$. Suppose that $q$ is bounded, $\operatorname{supp} q \subset B\left(0, r_{0}\right)$ and 0 is not an eigenvalue of $-\Delta+q$. Then there is a constant $\rho=\rho\left(r_{0}, d\right)$, such that for any $0 \leq j, 1 \leq p \leq p_{j}$ and $0 \leq k, 1 \leq q \leq p_{k}$, we have:

$$
\left|\left\langle\left(\Lambda_{q}-\Lambda_{0}\right) f_{j p}, f_{k q}\right\rangle\right| \leq \rho r_{0}^{\max (j, k)}\|q\|_{\infty}\left\|(-\Delta+q)^{-1}\right\|_{L^{2}}
$$

Proof. We fix the indices $j, p, k, q$. Consider the problem (1) with $f=f_{j p}$ and denote its solution by $u$. Also, denote $u_{0}(r, \omega)=r^{j} f_{j p}(\omega)$, the harmonic function with boundary value $f_{j p}$. Then $u-u_{0}$ has zero boundary value, so it is in the domain of $-\Delta+q$, and since $(-\Delta+q)\left(u-u_{0}\right)=-q u_{0}$ in $\Omega$, we obtain

$$
u-u_{0}=-(-\Delta+q)^{-1} q u_{0} .
$$

Since $\left\|u_{0}\right\|_{L^{2}\left(B\left(0, r_{0}\right)\right)}=\left(\int_{0}^{r_{0}} r^{2 j+d-1}\right)^{1 / 2}\left\|f_{j p}\right\|_{L^{2}\left(S^{d-1}\right)}=(2 j+d)^{-1 / 2} r_{0}^{j+d / 2}<r_{0}^{j}$ and $q$ has support in $B\left(0, r_{0}\right)$, we get :

$$
\left\|u-u_{0}\right\|_{L^{2}} \leq r_{0}^{j}\|q\|_{\infty}\left\|(-\Delta+q)^{-1}\right\|_{L^{2}}
$$

The function $v:=u-u_{0}$ is harmonic in $\Omega \backslash B\left(0, r_{0}\right)$ and equal to zero on $\partial \Omega$. We extend it to a function in $\left\{r_{0}<|x|<1 / r_{0}\right\}$, by putting $v(x)=-|x|^{2-d} v\left(|x|^{-2} x\right)$ for $1<|x|<1 / r_{0}$. Then $v$ is continuous with its first derivatives across $\partial \Omega$, hence $v$ is harmonic in the annulus $\left\{r_{0}<|x|<1 / r_{0}\right\}$. We can estimate the $L^{2}$-norm of $v$ in $\left\{1<|x|<\min \left(2,1 / r_{0}\right)\right\}$ by its $L^{2}$-norm in $\left\{r_{0}<|x|<1\right\}$. Then applying interior estimates for the derivative of $v$, we obtain

$$
\left\|\left.\frac{\partial v}{\partial \nu}\right|_{\partial \Omega}\right\|_{L^{2}(\partial \Omega)} \leq \rho\|v\|_{L^{2}\left(\left\{r_{0}<|x|<1\right\}\right)},
$$

with a constant $\rho$ depending on $r_{0}$ and $d$.

Combining (8) with (9) and taking into account that $\left(\Lambda_{q}-\Lambda_{0}\right) f_{j p}=\left.\frac{\partial v}{\partial \nu}\right|_{\partial \Omega}$, we obtain (6) for $j \geq k$. For $k>j$ we use the fact that $\Lambda_{q}^{*}=\Lambda_{\bar{q}}$ to swap the roles of $j$ and $k$. 


\section{A fat metric space and a thin metric space}

In this section we prove Theorem 1 and its corollary. All the numbers, function spaces, etc. are supposed real.

Definition 1 Let $(X, d)$ be a metric space and $\varepsilon>0$. We say that a set $Y \subset X$ is an $\varepsilon$-net for $X_{1} \subset X$ if for any $x \in X_{1}$ there is $y \in Y$ such that $d(x, y) \leq \varepsilon$. A set $Z \subset X$ is called $\varepsilon$-discrete if for any distinct $z_{1}, z_{2} \in Z$, we have $d\left(z_{1}, z_{2}\right) \geq \varepsilon$.

Lemma 2 Let $d \geq 2$ and $m>0$. For $\varepsilon, \beta>0$, consider the metric space

$$
X_{m \varepsilon \beta}=\left\{f \in C_{0}^{m}(B(0,1 / 2)):\|f\|_{\infty} \leq \varepsilon,\|f\|_{C^{m}} \leq \beta\right\}
$$

with the metric induced by $L^{\infty}$. Then there is a $\mu>0$ such that for any $\beta>0$ and $\varepsilon \in(0, \mu \beta)$, there is an $\varepsilon$-discrete set $Z \subset X_{m \varepsilon \beta}$ with at least $\exp \left(2^{-d-1}(\mu \beta / \varepsilon)^{d / m}\right)$ elements.

Proof. Take $\psi$ in $C_{0}^{\infty}\left(\mathbb{R}^{d}\right)$ with support in $(-1 / 2,1 / 2)^{d}$ and $\|\psi\|_{\infty}=1$. We put $\mu=\frac{1}{d^{m / 2}\|\psi\|_{C^{m}}}$. Denote $N=\left[\left(\frac{\mu \beta}{\varepsilon}\right)^{1 / m}\right]$. Then $\varepsilon<\mu \beta$ implies $\left(\frac{\mu \beta}{\varepsilon}\right)^{1 / m}>1$, so $N>2^{-1}\left(\frac{\mu \beta}{\varepsilon}\right)^{1 / m}$. The cube $\left(-\frac{1}{2 \sqrt{d}}, \frac{1}{2 \sqrt{d}}\right)^{d}$ is included in the ball $B(0,1 / 2)$. We divide it into $N^{d}$ smaller cubes of edge $\frac{1}{N \sqrt{d}}$. Let $y_{1}, \ldots, y_{N^{d}}$ be their centres. Then we take:

$$
Z=\left\{\varepsilon \sum_{j=1}^{N^{d}} \sigma_{j} \psi\left(N \sqrt{d}\left(x-y_{j}\right)\right) \mid \sigma_{j} \in\{0,1\} \text { for any } j\right\}
$$

This is contained in $X_{m \varepsilon \beta}$. Indeed, $\|\psi(a \cdot)\|_{C^{m}} \leq a^{m}\|\psi\|_{C^{m}}$ for $a \geq 1$ and we apply this for $a=N \sqrt{d} \leq\left(\frac{\beta}{\varepsilon\|\psi\|_{C^{m}}}\right)^{1 / m}$. Two distinct functions from $Z$ differ by at least one of the choices of $\sigma_{j}$, and in the corresponding cube one of them is zero and the other is $\left.\varepsilon \psi\left(N \sqrt{d}\left(x-y_{j}\right)\right)\right)$. Since $\|\psi\|_{\infty}=1$, we obtain that $Z$ is $\varepsilon$-discrete in the $L^{\infty}$ norm. The cardinality of the set $Z$ is $2^{N^{d}} \geq \exp \left(\left(2^{-1}(\mu \beta / \varepsilon)^{1 / m}\right)^{d} \log 2\right) \geq \exp \left(2^{-d-1}(\mu \beta / \varepsilon)^{d / m}\right)$. 
Consider an operator $A: H^{-s}\left(S^{d-1}\right) \rightarrow H^{s}\left(S^{d-1}\right)$. We denote its matrix elements in the basis $\left(f_{j p}\right)$ by $a_{j p k q}:=\left\langle A f_{j p}, f_{k q}\right\rangle$. Let $n_{l}$ be the number of 4-tuples of integers $(j, p, k, q)$, with $j, k \geq 0,1 \leq p \leq p_{j}, 1 \leq q \leq p_{k}$ and $\max (j, k)=l$. Then we have $n_{l}=2 p_{l} \sum_{j=0}^{l-1} p_{j}+p_{l}^{2} \leq 8(l+1)^{2 d-3}$. Using this we can estimate:

$$
\begin{aligned}
\|A\|_{H^{-s} \rightarrow H^{s}}^{2} & \leq\left\|\left(a_{j p k q}(1+j)^{s}(1+k)^{s}\right)\right\|_{H S}^{2} \\
& \leq \sum_{\substack{j \geq 0,1 \leq p \leq p_{j} \\
k \geq 0,1 \leq q \leq p_{k}}}(1+\max (j, k))^{4 s}\left|a_{j p k q}\right|^{2} \\
& \leq 16 \sup _{j, p, k, q}(1+\max (j, k))^{4 s+2 d-1}\left|a_{j p k q}\right|^{2}
\end{aligned}
$$

We have used $\sum_{j, p, k, q}(1+\max (j, k))^{-2 d+1}=\sum_{l=0}^{\infty} n_{l}(1+l)^{-2 d+1} \leq 8 \sum_{1}^{\infty} l^{-2} \leq 16$. We introduce the Banach space

$$
X_{s}:=\left\{\left(a_{j p k q}\right)\left|\left\|\left(a_{j p k q}\right)\right\|_{X_{s}}:=\sup _{j, p, k, q}(1+\max (j, k))^{2 s+d}\right| a_{j p k q} \mid<\infty\right\} .
$$

Let us denote $\Lambda_{q}-\Lambda_{0}$ by $\Gamma(q)$. Then $\Gamma(q)$ is defined for all bounded $q$ such that $-\Delta+q$ does not have the Dirichlet eigenvalue 0 . Denote by $B^{\infty}$ the unit ball of $L^{\infty}(B(0,1 / 2))$. We identify in the sequel an operator $A: H^{1 / 2}(\partial \Omega) \rightarrow$ $H^{-1 / 2}(\partial \Omega)$ with its matrix $\left(a_{j p k q}\right)$. The estimate $(11)$ shows that $\|A\|_{H^{-s} \rightarrow H^{s}} \leq$ $4\left\|\left(a_{j p k q}\right)\right\|_{X_{s}}$.

Lemma $3 \Gamma$ maps $B^{\infty}$ into $X_{s}$ for any $s$. There is a constant $0<\eta=\eta(s, d)$, such that for every $\delta \in\left(0, e^{-1}\right)$, there is a $\delta$-net $Y$ for $\Gamma\left(B^{\infty}\right)$ in $X_{s}$, with at most $\exp \left(\eta(-\log \delta)^{2 d-1}\right)$ elements.

Proof. We can suppose $s \geq 0$ as the assertion is stronger in this case. Let $K=\max (1, \rho(1 / 2, d))$, with $\rho(1 / 2, d)$ of Lemma 1 . For $q \in B^{\infty}$ we have $\|q\|_{\infty} \leq 1$ and $\left\|(-\Delta+q)^{-1}\right\| \leq\left(\lambda_{1}-1\right)^{-1}$, with $\lambda_{1}$ being the first eigenvalue of $-\Delta$ on $\Omega$. $\lambda_{1}$ is increasing with the dimension and for $d=2$ we have $\lambda_{1} \approx 5.783$. We obtain from (6) that $\left|a_{j p k q}\right| \leq K 2^{-\max (j, k)}$ for $\left(a_{j p k q}\right) \in \Gamma\left(B^{\infty}\right)$, hence $\left\|\left(a_{j p k q}\right)\right\|_{X_{s}} \leq \sup _{l}(1+l)^{2 s+d} K 2^{-l}<\infty$ for any $s$ and $d$, so the first assertion of the Lemma is proven. 
Let $l_{\delta s}$ be the smallest integer such that $(1+l)^{2 s+d} K 2^{-l} \leq \delta$ for any $l \geq l_{\delta s}$. Since $\log \delta^{-1} \geq 1$, we have $l_{s \delta} \leq C \log \delta^{-1}$, with $C$ depending only on $s$ and $d$. Denote $\delta^{\prime}=\left(1+l_{\delta s}\right)^{-2 s-d} \delta$. Consider the set

$$
Y_{\delta s}:=\delta^{\prime} \mathbb{Z} \bigcap[-K, K]
$$

Then $\left|Y_{\delta s}\right|=1+2\left[K / \delta^{\prime}\right] \leq C \delta^{-1}\left(\log \delta^{-1}\right)^{2 s+d}$. We set

$$
Y=\left\{\left(a_{j p k q}\right) \mid a_{j p k q} \in Y_{\delta s} \text { for } \max (j, k) \leq l_{\delta s}, a_{j p k q}=0 \text { otherwise }\right\}
$$

In order to prove that $Y$ is a $\delta$-net, let $\left(a_{j p k q}\right)$ be in $\Gamma\left(B^{\infty}\right)$. We construct an element $\left(b_{j p k q}\right)$ in $Y$, within $X_{s}$-distance $\delta$ from $\left(a_{j p k q}\right)$. If $\max (j, k) \leq l_{\delta s}$, we take $b_{j p k q}$ to be one of the closest elements of $Y_{\delta s}$ to $a_{j p k q}$. Since $\left|a_{j p k q}\right| \leq K$, this ensures $\left|a_{j p k q}-b_{j p k q}\right| \leq \delta^{\prime}$. If $j>l_{\delta s}$ or $k>l_{\delta s}$, we take $b_{j p k q}=0$. We have then $(1+\max (j, k))^{2 s+d}\left|a_{j p k q}-b_{j p k q}\right| \leq \delta$. For $\max (j, k)>l_{\delta s}$, this is true by the construction of $l_{\delta s}$, otherwise, by the choice of $\delta^{\prime}$. Then we get $\left\|\left(a_{j p k q}\right)-\left(b_{j p k q}\right)\right\|_{X_{s}} \leq \delta$ as required.

It remains to count the elements of $Y$. There are exactly $\left|Y_{\delta s}\right|^{n_{\delta s}}$ elements, where $n_{\delta s}=\sum_{j=0}^{l_{\delta s}} n_{j} \leq 8\left(1+l_{\delta s}\right)^{2 d-2}$. Then using again the fact that $\log \delta^{-1} \geq$ 1 , and the estimates for $\left|Y_{\delta s}\right|$ and $l_{\delta s}$ :

$$
\begin{aligned}
|Y| & \leq\left(C \delta^{-1}\left(\log \delta^{-1}\right)^{2 s+d}\right)^{8\left(1+C^{\prime} \log \delta^{-1}\right)^{2 d-2}} \\
& \leq \exp \left(C^{\prime \prime} \log \delta^{-1}\left(C^{\prime} \log \delta^{-1}\right)^{2 d-2}\right) \\
& \leq \exp \left(C^{\prime \prime \prime}\left(\log \delta^{-1}\right)^{2 d-1}\right) .
\end{aligned}
$$

Proof of Theorem 1. If the set $X_{m \varepsilon \beta}$ has an $\varepsilon$-discrete set $Z$ and $\Gamma\left(X_{m \varepsilon \beta}\right)$ has a $\delta$-net $Y$ in $X_{s}$, and $|Z|>|Y|$, then there are two points in $Z$ with images under $\Gamma$ in the same $X_{s} \delta$-ball centered at a point of $Y$, so we get $\| \Lambda_{q_{1}}-$ $\Lambda_{q_{2}}\left\|_{H^{-s} \rightarrow H^{s}} \leq 4\right\| \Gamma\left(q_{1}\right)-\Gamma\left(q_{2}\right) \|_{X_{s}} \leq 8 \delta$ for some $q_{1}, q_{2}$ with $\left\|q_{1}-q_{2}\right\|_{\infty} \geq \varepsilon$. We take $8 \delta=\exp \left(-\varepsilon^{\frac{-d}{(2 d-1) m}}\right)$, which is the right hand of (5). Since $X_{m \varepsilon \beta} \subset B^{\infty}$ for $\varepsilon \leq 1$, the set $Y$ constructed in Lemma 3 is also a $\delta$-net for $\Gamma\left(X_{m \varepsilon \beta}\right)$. It remains to choose $\beta$ such that $X_{m \varepsilon \beta}$ has an $\varepsilon$-discrete set $Z$ with $|Z|>|Y|$. By Lemma $3,|Y| \leq \exp \left(\eta\left(\log 8+\varepsilon^{-\frac{d}{(2 d-1) m}}\right)^{2 d-1}\right) \leq \exp \left(4^{2 d-1} \eta \varepsilon^{-d / m}\right)$. If $\beta>\varepsilon / \mu$, 
we can apply Lemma 2 and get $|Z| \geq \exp \left(2^{-d-1}(\beta \mu / \varepsilon)^{d / m}\right)$. Then choosing $\beta>\mu^{-1} \max \left(1,2^{5 m} \eta^{m / d}\right)$ the desired inequality $|Z|>|Y|$ is implied by the above two inequalities.

Proof of the corollary. We use the reduction of the problem (2) to (1). The conductivities $\gamma_{1}, \gamma_{2}$ are sought in the set $\tilde{X}_{m \varepsilon \beta}:=1+X_{m \varepsilon \beta}$ (see (10)). Since each $\gamma \in \tilde{X}_{m \varepsilon \beta}$ is 1 in a neighborhood of $\partial \Omega$, we have $\tilde{\Lambda}_{\gamma}=\Lambda_{q}$, with $q=\gamma^{-1 / 2} \Delta \gamma^{1 / 2}$. As in Lemma 2 , we can construct an $\varepsilon$-discrete set $\tilde{Z} \subset \tilde{X}_{m \varepsilon \beta}$ with $\gamma \geq 1$ for all $\gamma \in \tilde{Z}$ and

$$
|\tilde{Z}| \geq \exp \left(C(\beta / \varepsilon)^{d / m}\right)
$$

with $C>0$ depending only on $m$ and $d$. Suppose from now on that $\gamma \in \tilde{Z}$, and $q$ is the corresponding potential. From $\gamma \geq 1$ we get $-\Delta \leq$ $-\operatorname{div} \gamma \nabla$ as operators in $L^{2}(\Omega)$, hence $\left\|(-\operatorname{div} \gamma \nabla)^{-1}\right\|_{L^{2}} \leq 1 / \lambda_{1}$. We have $-\Delta+q=\gamma^{-1 / 2}(-\operatorname{div} \gamma \nabla) \gamma^{-1 / 2}$ and $\gamma \leq 2$, so we obtain $\left\|(-\Delta+q)^{-1}\right\|_{L^{2}} \leq$ $2 / \lambda_{1}<1$. The potential satisfies $\|q\|_{\infty} \leq C_{1}\|\gamma\|_{C^{2}}^{2} \leq C_{1} \beta^{2}$, because $m \geq 2$. Let $\delta=\frac{1}{8} \exp \left(-\varepsilon^{\frac{-d}{(2 d-1) m}}\right)$ as in the proof of Theorem 1. Taking $K=\max \left(1, \rho(1 / 2, d) C_{1} \beta^{2}\right) \leq C^{\prime} \beta^{2}$ in the proof of Lemma 3 , we get a $\delta$-net $\tilde{Y}$ for the image under $\Gamma$ of the set of potentials corresponding to conductivities in $\tilde{Z}$, with

$$
|\tilde{Y}| \leq \exp \left(C^{\prime \prime} \varepsilon^{-d / m} \log \beta^{2}\right) .
$$

Comparing (12) with (13) we see that for $\beta$ big enough, we get $|\tilde{Y}|<|\tilde{Z}|$ for any $\varepsilon \in(0,1)$. Then we reason as in the proof of Theorem 1 .

\section{$5 \quad$ Further results}

We give first an explicit counterexample with complex potential. Let us consider cylindrical variables $\left(r_{1}, \theta, x^{\prime}\right) \in \mathbb{R}_{+} \times \mathbb{R} / 2 \pi \mathbb{Z} \times \mathbb{R}^{d-2}$, with $x^{\prime}=$ $\left(x_{3}, \ldots, x_{d}\right), r_{1} \cos \theta=x_{1}$ and $r_{1} \sin \theta=x_{2}$. Take $\phi \in C_{0}^{\infty}\left(\mathbb{R}^{2}\right)$ with support in $B(0,1 / 2) \cap\left\{x_{1}>1 / 4\right\}$ and with $\|\phi\|_{\infty}=1$.

Theorem 2 For $m>0$ and integer $n>0$, define the complex potential

$$
q_{n m}(x)=n^{-m} e^{i n \theta} \phi\left(r_{1},\left|x^{\prime}\right|\right) .
$$


Then $\left\|q_{m n}\right\|_{\infty}=n^{-m}$ and for every $d$ and $m$ there are constants $c, c^{\prime}>0$ such that $\left\|q_{m n}\right\|_{C^{m}} \leq c$ and $\left\|\Lambda_{q_{m n}}-\Lambda_{0}\right\|_{L^{2}} \leq c^{\prime} 2^{-n / 2}$ for every $n$.

In a sense, this is stronger than Theorem 1. Indeed, if we take $\varepsilon=n^{-m}$, we obtain (5) with $\exp \left(-C \varepsilon^{-1 / m}\right)$ in the right hand side. An explicit real valued counterexample should be difficult to find. This is due to the nonlinearity of the map $q \rightarrow \Lambda_{q}$. Roughly speaking, we need to consider powers of $q$ (in fact, they are intertwined with $(-\Delta)^{-1}$, see (14) below). The powers $q_{m n}^{k}, k \geq 1$, have no component in the spherical harmonics of degree $<n$. By contrast, if $q$ is real valued, then $q^{2}$ has already a non-negligible component of degree 0 , and we cannot make use of Lemma 1.

Proof of Theorem 2. First, $q_{m n}$ has support in $\left\{r_{1}>1 / 4\right\}$, hence $\left\|q_{m n}\right\|_{C^{m}}$ remains bounded as $n \rightarrow \infty$. Since $\left\|q_{m n}\right\|_{\infty} \leq 1<\lambda_{1}$, we can express the inverse of $\left(-\Delta+q_{m n}\right)^{-1}$ in (7) using a Neumann series to obtain, with the notations of the proof of Lemma 1 :

$$
u-u_{0}=\sum_{j=1}^{\infty}\left((-\Delta)^{-1} q_{m n}\right)^{j} u_{0}
$$

For any integer $k$, let $L_{k}^{2}(\Omega):=\left\{f \in L^{2}(\Omega) \mid e^{-i k \theta} f\right.$ does not depend on $\left.\theta\right\}$. The spaces $\left\{L_{k}^{2}(\Omega)\right\}_{k \in \mathbb{Z}}$ are orthogonal and span $L^{2}(\Omega)$. The potential $q_{m n}$, as a multiplication operator, maps $L_{k}^{2}(\Omega)$ into $L_{k+n}^{2}(\Omega)$. On the other hand, $L_{k}^{2}(\Omega)$ are invariant for $-\Delta$ hence they are invariant for $(-\Delta)^{-1}$.

In a similar way we introduce $L_{k}^{2}(\partial \Omega)$. Suppose $n \geq 1$ and take $n^{\prime}=\left[\frac{n-1}{2}\right]$. We notice that the harmonics of degree at most $n^{\prime}$ on $\partial \Omega$ are included in the direct sum $\bigoplus_{k=-n^{\prime}}^{n^{\prime}} L_{k}^{2}(\partial \Omega)$. We can see this expressing them as polynomials of $z, \bar{z}, x^{\prime}$, with $z=x_{1}+i x_{2}=r_{1} e^{i \theta}$.

We claim that $\left\langle\left(\Lambda_{q_{m n}}-\Lambda_{0}\right) f_{j p}, f_{k q}\right\rangle=0$ for $j, k \leq n^{\prime}$. Supposing this true, we apply Lemma 1 taking $a<1 / 2$ such that $\operatorname{supp} q_{m n} \subset B(0, a)$ and we obtain, in a way similar to (11),

$$
\left\|\Lambda_{q_{m n}}-\Lambda_{0}\right\|_{L^{2}} \leq\left\|\Lambda_{q_{m n}}-\Lambda_{0}\right\|_{H S} \leq \sum_{j=n^{\prime}+1}^{\infty} \rho 8(j+1)^{2 d-3} a^{j} \leq c^{\prime} 2^{-n / 2}
$$


To prove the claim, suppose $j, k \leq n^{\prime}$. Then $f_{j p} \in \bigoplus_{k=-n^{\prime}}^{n^{\prime}} L_{k}^{2}(\partial \Omega)$ and $u_{0}(r, \omega)=$ $r^{j} f_{j p}(\omega) \in \bigoplus_{k=-n^{\prime}}^{n^{\prime}} L_{k}^{2}(\Omega)$. Then we have $\left((-\Delta)^{-1} q_{m n}\right)^{l} u_{0} \in \bigoplus_{k=n l-n^{\prime}}^{n l+n^{\prime}} L_{k}^{2}(\Omega)$, so, by (14), $u-u_{0} \in \bigoplus_{k=n-n^{\prime}}^{\infty} L_{k}^{2}(\Omega)$. This shows that $\left(\Lambda_{q_{m n}}-\Lambda_{0}\right) f_{j p}=\left.\frac{\partial\left(u-u_{0}\right)}{\partial \nu}\right|_{\partial \Omega} \in$ $\bigoplus_{k=n-n^{\prime}}^{\infty} L_{k}^{2}(\partial \Omega)$ and since $n-n^{\prime}>n^{\prime}$ and $f_{k q} \in \bigoplus_{k=-n^{\prime}}^{n^{\prime}} L_{k}^{2}(\partial \Omega)$, the claim is proven.

While this paper was in preparation, professor Isakov suggested that even radial potentials might give counterexamples to stability. An analog of Theorem 1 can be proved for such potentials, obtaining $\frac{-1}{(2 d-1) m}$ as the exponent of $\varepsilon$ in $(5)$, instead of $\frac{-d}{(2 d-1) m}$. This can be improved to $\frac{-1}{2 m}$ using the following

Lemma 4 An operator $A$ acting on $L^{2}\left(S^{d-1}\right)$, which commutes with rotations, has the matrix elements $a_{j p k q}=a_{j} \delta_{j k} \delta_{p q}$, with $\delta_{j k}$ the Kronecker symbol.

Considering only radial potentials has the effect of reducing the size of the $\varepsilon$ discrete set $Z$ that we can construct in Lemma 2 to $\exp \left(C(\beta / \varepsilon)^{1 / m}\right)$. However, the size of the $\delta$-net of Lemma 3 is also reduced to $\exp \left(\eta\left(\log \delta^{-1}\right)^{2}\right)$, because by Lemma 4, the number of relevant dimensions for the set $Y$ is reduced to $1+l_{\delta s}$.

Proof of Lemma 4. If $R$ is a rotation, denote its action in $L^{2}\left(S^{d-1}\right)$ by $R^{*} f(x)=f(R x)$. Then $R^{*} A=A R^{*}$ is equivalent to $K_{A}(R x, y)=$ $K_{A}\left(x, R^{-1} y\right)$, where $K_{A}$ is the Schwartz kernel of the operator $A$. This implies that $K_{A}(x, y)=F(\operatorname{dist}(x, y))$ for a certain $F$. Let $\Pi_{m}, m \geq 0$ integer, be the orthogonal projection in $L^{2}\left(S^{d-1}\right)$ on the space of harmonics of degree $m$. Then $\Pi_{m}$ commutes with rotations and applying the same argument as above, it has a Schwartz kernel $P_{m}(\operatorname{dist}(x, y))$. Let $e_{d}=(0, \ldots, 0,1)$ be the North pole, and $f_{m}(x):=P_{m}\left(\operatorname{dist}\left(x, e_{d}\right)\right)=\Pi_{m} \delta_{e_{d}}(x)$. Then $f_{m}$ is a spherical harmonic of degree $m$, and modulo a constant, it is the only one which is only a function of $\operatorname{dist}\left(x, e_{d}\right)$. Indeed, according to the parity of $m$, such a function is either odd or even. If there were two linearly independent such functions, we would be able to construct a non-zero spherical harmonic of degree $m$ which vanishes with its first order derivatives on $S^{d-1} \cap\left\{x_{d}=0\right\}$, and 
this is not possible. Expanding $F\left(\operatorname{dist}\left(x, e_{d}\right)\right)$ in spherical harmonics we get $F=\sum_{m=0}^{\infty} a_{m} P_{m}$, since all components need to be invariant under rotations that have $e_{d}$ as a fixed point. It follows that $A=\sum_{m=0}^{\infty} a_{m} \Pi_{m}$.

Aknowledgements. This work was supported by the EPSRC grant GRM14463. I would like to thank Y. Kurylev for suggestions which led to many improvements, and G. Alessandrini, J.A. Barceló, V. Isakov, A. Ruiz and G. Uhlmann for stimulating discussions.

\section{References}

[1] G. Alessandrini, Stable determination of conductivity by boundary measurements, Appl. Anal. 27, (1988), 153-172.

[2] G. Alessandrini, Singular solutions of elliptic equations and the determination of conductivity by boundary measurements, J. Diff. Eq. 84, (1990), $252-272$.

[3] G. Alessandrini, Determining conductivity by boundary measurements, the stability issue, R. Spigler (ed.), Applied and Industrial Mathematics, Kluwer, 1991, 317-324.

[4] G. Alessandrini, Examples of instability in inverse boundary-value problems, Inverse Problems 13 (1997), 887-897.

[5] R. Brown and G. Uhlmann, Uniqueness in the inverse conductivity problem with less regular conductivities, Comm. Partial Differential Equations 22 (1997), 1009-1027.

[6] J.A. Barceló, B. Barceló, A. Ruiz, Stability of the inverse conductivity problem in the plane for less regular conductivities, to appear.

[7] A.P. Calderón, On an inverse boundary value problem, Seminar on Numerical Analysis and its applications to Continuum Physics, Soc. Brasil. Mat., Rio de Janeiro, (1980), 65-73. 
[8] V. Isakov, Inverse Problems for Partial Differential Equations, Applied Mathematical Sciences 127, Springer Verlag, 1998.

[9] R. Kohn and M. Vogelius, Determining conductivity by boundary measurrements, Comm. Pure App. Math. 37 (1984), 289-298.

[10] L. Liu, Stability estimates for the two dimensional inverse conductivity problem, PhD Thesis, Dep. of Mathematics, University of Rochester, New York, (1997).

[11] A. Nachman, Inverse scattering at fixed energy, Proceedings of the tenth Internation Congress on Mathematical Physics, Leipzig, 1991, Springer Verlag, 434-441.

[12] A. Nachman, Global uniqueness for a two-dimensional inverse boundary value problem, Ann. of Math. 142 (1995), 71-96.

[13] Z. Sun and G. Uhlmann, Generic uniqueness for an inverse boundary problem, Duke Math. J. 62 (1991), 131-155.

[14] J. Sylvester and G. Uhlmann, A global uniqueness theorem for an inverse boundary value problem, Ann. of Math. 125 (1987), 153-169.

[15] J.Sylvester and G. Uhlmann, Inverse boundary value problems at the boundary - continuous dependence, Comm. Pure Appl. Math. 41 (1988), $197-221$. 